\title{
The Changing Spectrum of Surgically Treated Cystic Neoplasms of the Pancreas
}

\author{
Jennifer K. Plichta, Jacqueline A. Brosius, Sam G. Pappas, \\ Gerard J. Abood, and Gerard V. Aranha
}

Department of Surgery, Loyola University Health System, Maywood, IL 60153, USA

Correspondence should be addressed to Gerard V. Aranha; garanha@lumc.edu

Received 22 July 2014; Revised 15 February 2015; Accepted 19 March 2015

Academic Editor: Richard Charnley

Copyright (C) 2015 Jennifer K. Plichta et al. This is an open access article distributed under the Creative Commons Attribution License, which permits unrestricted use, distribution, and reproduction in any medium, provided the original work is properly cited.

\begin{abstract}
Introduction. While the incidence of pancreatic cystic lesions has steadily increased, we sought to evaluate the changes in their surgical management. Methods. Patients with pancreatic cystic lesions who underwent surgical resection from 2003 to 2013 were identified. Clinicopathologic factors were analyzed and compared to a similar cohort from 1992 to 2002. Results. There were 134 patients with pancreatic cystic lesions who underwent surgical resection from 2003 to 2013, compared to 73 from 1992 to 2002 . The most common preoperative imaging was a CT scan, although 66\% underwent EUS and 63\% underwent biopsy. Pathology included 18 serous, 47 mucinous, 11 pseudopapillary, and 58 intraductal papillary mucinous neoplasms (IPMN). In comparing cohorts, there were significantly fewer serous lesions and more IPMN. Postoperative complication rates were similar, and perioperative mortality rates were comparable. Conclusion. There has been a dramatic change in surgically treated pancreatic cystic tumors over the past two decades. Our data suggests that the incorporation of new imaging and diagnostic tests has led to greater detection of cystic tumors and a decreased rate of potentially unnecessary resections. Therefore, all patients with cystic pancreatic lesions should undergo a focused CT-pancreas, and an EUS biopsy should be considered, in order to best select those that would benefit from surgical resection.
\end{abstract}

\section{Introduction}

Neoplasms comprise $50 \%$ of cystic lesions of the pancreas [1]. They are divided into four main subtypes: serous cystic neoplasms (SCN), mucinous cystic neoplasms (MCN), solid pseudopapillary neoplasms (SPN), and intraductal papillary mucinous neoplasms (IPMN). Most frequently, these neoplasms are found incidentally, and the number of patients diagnosed with a neoplasm by imaging done for an unrelated reason is $>2 \%$ [2]. The rise in routine use of computed tomography (CT) scans and magnetic resonance imaging (MRI) has led to an increase in the diagnosis of asymptomatic cystic neoplasms [3].

In general, IPMN, mucinous cysts, and SPN are removed surgically, given their significant potential for malignant transformation. Serous cystadenomas, in contrast, have a negligible rate of malignant transformation, although these lesions are more likely to be larger and cause symptoms due to mass effect [4]. It is considered standard of care to resect symptomatic serous cystadenomas that cause significant morbidity. However, the decision to resect asymptomatic masses has been more controversial, and management varies greatly by institution [4]. In the past, a more aggressive approach has been utilized for serous cystadenomas, due to concern for misdiagnosis based on standard, older imaging [4].

Pancreas-specific CT, developed in the mid-1990s, is a multidetector CT (MDCT) that allows for visualization of arterial, pancreatic, and portal venous phases and drastically enhances the diagnostic accuracy of pancreatic tumors [5]. The introduction of this technology along with endoscopic ultrasound- (EUS-) guided biopsies (fine needle aspiration FNA) has provided us with new ways to confirm pathology before operative intervention is undertaken. These advancements in imaging and technology have subsequently influenced the management of these neoplasms.

The objective of this study was to review our institution's experience with pancreatic cystic lesions and to characterize 
their presentation, diagnosis, and perioperative management. In order to determine how the spectrum of surgically treated cystic neoplasms of the pancreas may have changed over the years, we compared these patients to a previous study conducted by our institution from 1992 to 2002 [6].

\section{Methods}

Through a retrospective chart review, patients with pancreatic cystic lesions who underwent surgical resection from 2003 to 2013 at Loyola University Medical Center were identified (modern cohort). Patients with a prior history of pancreatic cancer were excluded. Clinicopathologic factors were assessed from the medical record, including clinical presentation, preoperative evaluation/imaging, surgeries performed, pathologic results, postoperative complications, and mortalities. The Social Security Death Index was utilized to determine current living status of patients in the modern cohort.

In the preoperative setting, a CT scan was typically the initial imaging performed. For patients with a contrast allergy or nonspecific findings on CT, an MRI was obtained. The main criterion for doing an EUS in the modern cohort was to get an accurate diagnosis of serous and mucinous tumors. If CT clearly showed a SPN or main duct IPMN, then EUS was not performed. However, EUS was done for asymptomatic branch duct IPMN to evaluate it for possible malignant features. In general, when an EUS was performed at our institution in the modern cohort, cyst fluid was sent for mucin, CEA (carcinoembryonic antigen), amylase, and cytology. Cysts that were high in amylase with no mucin or CEA and negative cytology were considered to be pseudocysts and were excluded. Fluids that had no mucin, low amylase, and low CEA were considered serous and were operated upon only if symptomatic. Cysts high in mucin with high CEA and atypical or malignant cytology went to surgery.

Select results were compared to a similar cohort of patients from 1992 to 2002 (data previously collected [6]). Statistical analyses were conducted using Stata 10.0 (StataCorp, College Station, TX). Categorical variables were analyzed using Chi-squared $\left(\chi^{2}\right)$ tests, and continuous variables were analyzed using Mann-Whitney $U$ tests or Student's $t$-tests. Statistical significance was defined as $P \leq 0.05$ (2-sided). This study was approved by the Loyola University Health Systems Institutional Review Board.

\section{Results}

From 2003 to 2013, there were 134 patients with pancreatic cystic lesions who underwent surgical resection (modern cohort). The median age of the modern cohort was 66 years (range 18-88 years old). The historic cohort was comprised of patients who underwent surgical resection from 1992 to 2002 and included 73 patients. Patients were predominately females in both populations (67\% versus 67\%). The most common presenting symptom was abdominal pain $(48 \%$, $n=62)$, which was less common than observed in the historic cohort $(64 \%, P=0.013)$. However, the same number

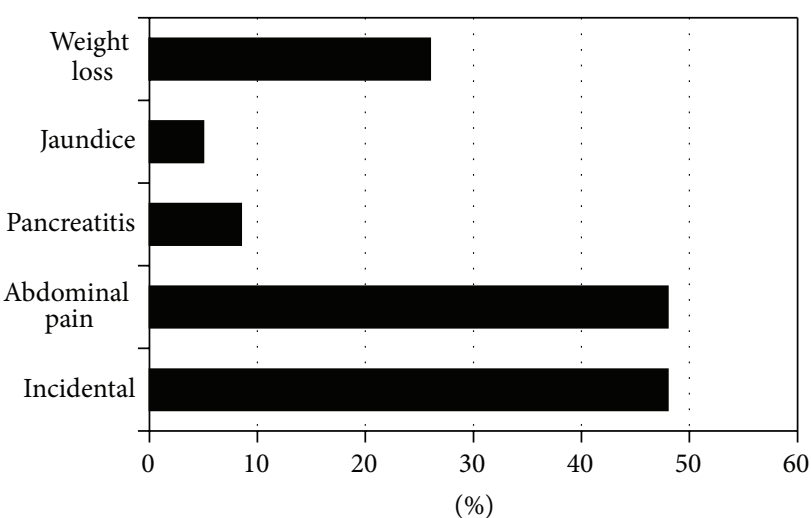

FIGURE 1: The most common clinical presentations in patients with pancreatic cystic lesions who underwent surgical resection from 2003 to 2013.

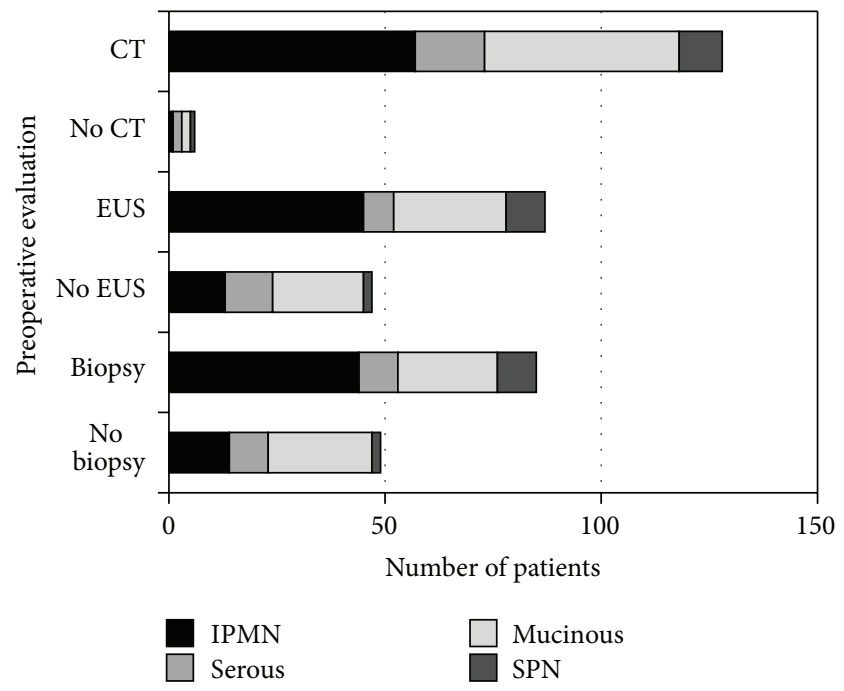

FIGURE 2: Preoperative evaluation of the modern cohort, including CT scans, endoscopic ultrasounds, and biopsy in select patients, stratified by the type of pancreatic cystic neoplasm. CT: computed tomography scan; EUS: endoscopic ultrasound.

of lesions in the modern cohort was found incidentally on imaging $(48 \%, n=62)$. Other common clinical presentations for the modern cohort included 34 with weight loss, 11 with pancreatitis, and 7 with jaundice (Figure 1).

Prior to surgery, the majority of recent patients had a CT scan (98\%, $n=128)$, while only $66 \%$ underwent EUS $(n=87)$ and $63 \%$ underwent biopsy $(n=85)$; the data is summarized in Figure 2 and is stratified by the type of cystic neoplasm. This data (preoperative imaging and biopsy) is not available in our records for the historic cohort, and we were unable to collect this data retrospectively. For the patients who underwent a biopsy (or fine needle aspiration), the indications for surgery included 14 patients with malignant lesions, 25 patients with atypical cells, 13 patients with mucin \pm , an elevated CEA, and 4 patients with specific indications including (1) main duct IPMN; (2) an elevated CA19-9; (3) 


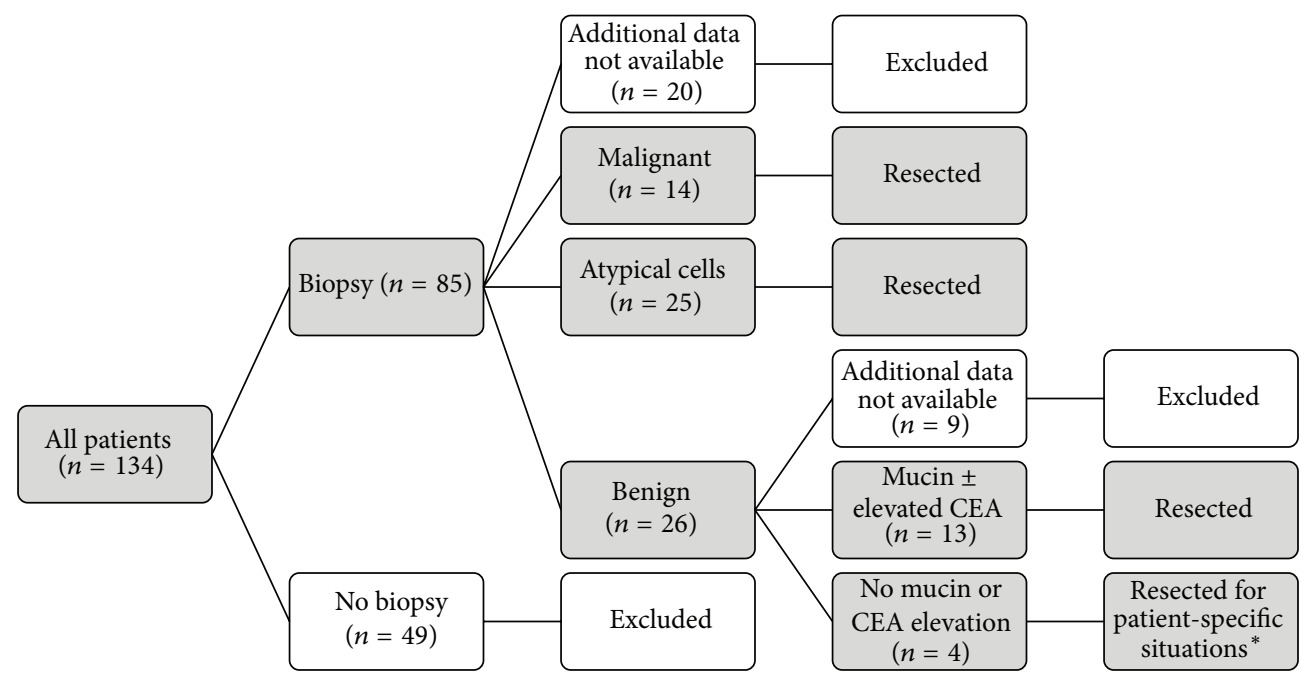

FIGURE 3: Results of preoperative biopsies and the algorithm for deciding when to operate. ${ }^{*}$ The four patients resected who did not meet standard biopsy criteria were for (1) main duct IPMN; (2) elevated serum CA19-9; (3) two separate pancreatic tumors in a patient with Von Hippel-Lindau disease (one serous and one pancreatic neuroendocrine tumor); and (4) patient symptoms. IPMN: intraductal papillary mucinous neoplasms; CA: cancer antigen; CEA: carcinoembryonic antigen.



Male

Female

FIGURE 4: Comparison of pancreatic cystic lesions by gender in the modern cohort; ${ }^{*} P<0.05$. SPN: solid pseudopapillary neoplasms; IPMN: intraductal papillary mucinous neoplasms.

two separate pancreatic tumors in a patient with Von HippelLindau disease (one serous, one pancreatic neuroendocrine tumor); and (4) patient symptoms. These results are outlined in Figure 3. The results of the biopsy were not available in 29 patients, as some patients were referred to our institution from an outside hospital and the records available were limited.

The main pathologic subtypes in the modern cohort included 18 serous lesions, 47 mucinous lesions, 11 SPN, and 58 IPMN. Most of the lesions were more common in females (serous $P=0.018$, mucinous $P=0.000$, and SPN $P=0.035$ ), while IPMN lesions were similarly distributed (Figure 4). Malignancy was noted in $17 \%$ of the mucinous lesions and
TABLE 1: Types of surgeries performed based upon the pathology of the pancreatic cystic lesions.

\begin{tabular}{lccccc}
\hline Type of tumor & PD & DP & CP & TP & Other \\
\hline SCN & 9 & 8 & 0 & 0 & 1 \\
Mucinous (MCN) & 17 & 26 & 1 & 1 & 2 \\
$\quad$ Cystadenoma & 12 & 23 & 1 & 1 & 2 \\
Cystadenocarcinoma & 5 & 3 & 0 & 0 & 0 \\
SPN & 3 & 8 & 0 & 0 & 0 \\
IPMN & 33 & 22 & 3 & 0 & 0 \\
\hline Total & 62 & 64 & 4 & 1 & 3 \\
\hline
\end{tabular}

SCN: serous cystic neoplasms; MCN: mucinous cystic neoplasms; SPN: solid pseudopapillary neoplasms; IPMN: intraductal papillary mucinous neoplasms; PD: pancreaticoduodenectomy; DP: distal pancreatectomy; CP: central pancreatectomy; TP: total pancreatectomy.

$38 \%$ of the IPMN. Compared to the historic cohort, there were significantly fewer serous lesions (13\% versus 36\%, $P=$ 0.0002 ) and more IPMN (43\% versus $25 \%, P=0.008)$. There was no difference in the incidence of mucinous lesions or SPN (Figure 5).

The majority of patients in the modern cohort underwent pancreaticoduodenectomy $(n=62)$ or distal pancreatectomy $(n=64)$. In addition, there were 4 central pancreatectomies, 1 total pancreatectomy, and 3 other procedures (Table 1). In the historic cohort, the most common surgical resection was also the distal pancreatectomy $(59 \%, n=43)$; however, pancreaticoduodenectomies were significantly more common in the modern cohort $(27 \%$ versus $46 \%, P=0.008$; Figure 6). Postoperatively, the most common complications in the modern cohort were wound infections ( $n=7)$, intraabdominal abscesses $(n=7)$, and urinary tract infections $(n=6)$. Other complications included 5 pancreatic fistulas, 5 with delayed gastric emptying, 3 gastrointestinal bleeds, and 


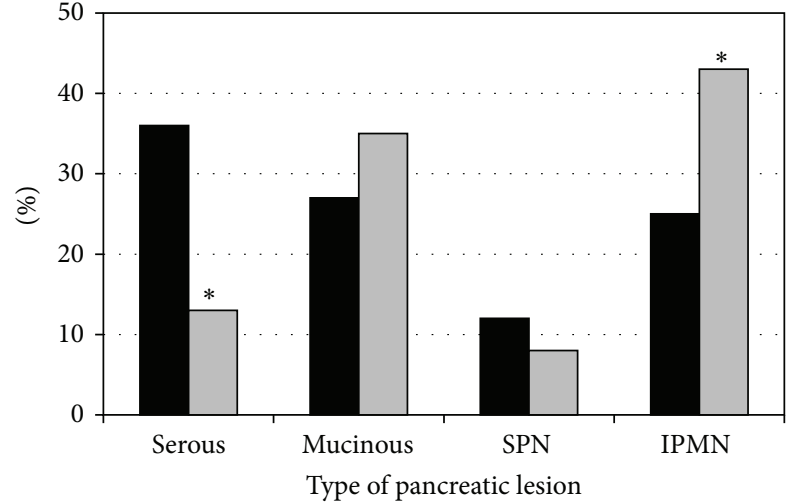

$1992-2002$

2003-2013

FIgURE 5: Comparison of pancreatic cystic lesions by patient cohort; ${ }^{*} P<0.01$. SPN: solid pseudopapillary neoplasms; IPMN: intraductal papillary mucinous neoplasms.

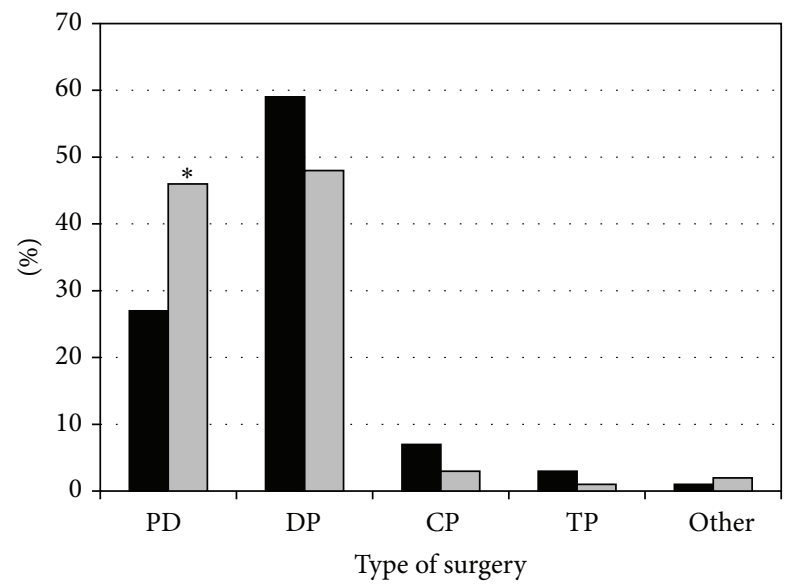

1992-2002

2003-2013

FIGURE 6: Comparison of surgeries performed by patient cohort; ${ }^{*} P<0.01$. PD: pancreaticoduodenectomy; DP: distal pancreatectomy; CP: central pancreatectomy; TP: total pancreatectomy.

2 pneumonias (Figure 7). Overall complication rates between the modern and historic cohorts were similar (32\% versus $27 \%, P=0.42$ ), while pancreatic fistula rates were slightly less common in the modern cohort (4\% versus $10 \%, P=0.085$ ). Perioperative mortality rates were comparable between both cohorts ( $3 \%$ versus $4 \%, P=0.67$; Figure 8 ).

\section{Discussion}

The increasing use of multidetector computed tomography (MDCT), magnetic resonance imaging (MRI), and endoscopic ultrasound (EUS) has specifically resulted in the increased recognition of pancreatic cystic lesions [7]. While some of these lesions are associated with malignancy, many of the lesions are asymptomatic at the time of diagnosis and benign in pathology. This has led to controversy in the

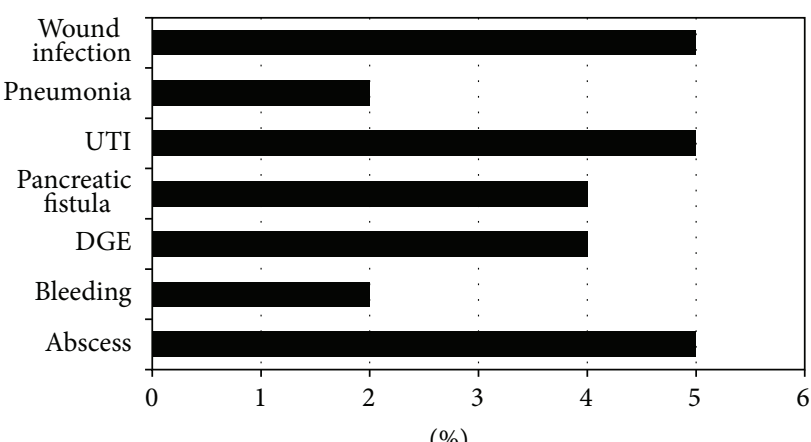

(\%)

FIGURE 7: Most common postoperative complications. UTI: urinary tract infection; DGE: delayed gastric emptying.

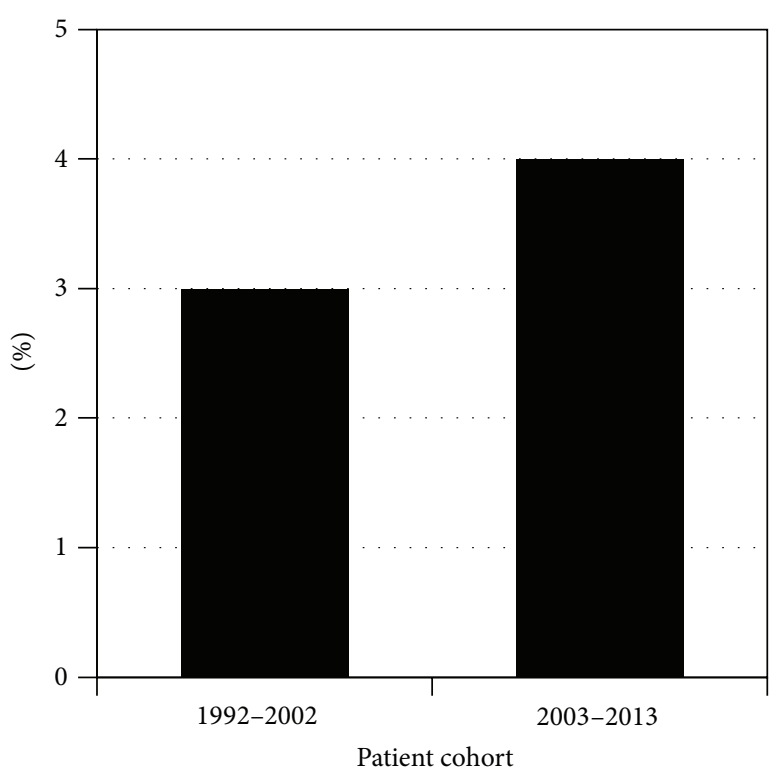

Figure 8: Comparison of mortalities by patient cohort.

surgical management of cystic pancreatic lesions [8]. Cystic lesions of the pancreas may be classified into neoplasms and pseudocysts [7]. The most common cystic neoplasms of the pancreas are serous cystic neoplasms (SCN), mucinous cystic neoplasms (MCN), solid pseudopapillary neoplasms (SPN), and intraductal papillary mucinous neoplasms (IPMN) [6].

We previously published our experience with the surgical management of cystic neoplasms between January 1992 and September 2002 [6]. In that decade, the most common cystic tumors surgically treated were SCN followed by MCN and both cystadenomas and cystadenocarcinomas, followed by IPMN, with SPN being the least common. Since that time, we and others $[6,9]$ have noted a change in the indications for surgery in pancreatic cystic neoplasms. At present, the most common indication for resection of a pancreatic cystic lesion is suspicion of malignancy, typically in IPMN and MCN. The prevalence of SCN has remained stable, while surgery for SCN has decreased dramatically. Why have all of these shifts occurred? The reasons are threefold: (1) SCN are often asymptomatic; (2) the incidence of malignancy 
in these tumors is quite low [10-13]; (3) the incidence of invasive carcinoma in IPMN is $15 \%$ at initial diagnosis and is cumulative over time [14]. Furthermore, improvements in MDCT, MRI, and EUS with cyst fluid analysis have helped differentiate the subtypes of cystic tumors prior to surgical treatment [15-17]. In a study by Lee et al., three radiologists were asked to interpret MDCTs and MRIs of 63 patients with cystic tumors of the pancreas, and the ability of each to predict malignancy was $77.8 \%, 73 \%$, and $73 \%$. These predictions were the same whether it was MDCT or MRI. The combination of MRI and MDCT was not significantly better but may merit future investigation [15].

The use of EUS in addition to CT (or MRI) has been recently evaluated by Khashab et al. [18]. This study evaluated 154 patients who underwent EUS and subsequent surgical resection and compared the diagnostic yield of the various imaging modalities. After CT or MRI, EUS increased the rate of correctly identifying neoplastic cysts by $36 \%$ and $54 \%$, respectively, and thus appears to be a useful adjunct in the preoperative evaluation of patients with cystic neoplasms. In the modern cohort of our patients, there were slightly more patients with serous lesions that did not undergo preoperative evaluation with EUS, which may suggest that the addition of this study may have yielded a more accurate diagnosis preoperatively and possibly avoided surgical resection in select cases. Overall, the decreased rate of surgical resection for serous lesions is likely related to the increased use of CT scans and confirmation by EUS when necessary.

Cyst fluid analysis from EUS has also demonstrated utility in diagnosing SCN $[13,19,20]$. In one study at Indiana University Medical Center, analysis of the cyst fluid using VEGF-A correlated with pathologic diagnosis [13]. For SCN specifically, a high level of VEGF-A $(8500 \mathrm{pg} / \mathrm{mL})$ had a sensitivity of $100 \%$ and specificity of $97 \%$. With a cutoff of $200 \mathrm{pg} / \mathrm{mL}$, VEGF-C identified SCN with 100\% sensitivity and $90 \%$ specificity. Another study from Massachusetts General Hospital demonstrated a low CEA level and a low amylase level in SCN lesions [19]. Therefore, the surgical intervention in SCN today is limited to those patients who are symptomatic, those who have large tumors, and those who have enlarging lesions. Our policy on asymptomatic SCN is to get a MDCT at six-month intervals for two years and then yearly for a period of five years.

Currently, the major controversy surrounding cystic tumors of the pancreas is related to IPMN. IPMN lesions can be classified into 3 subtypes: (1) main duct IPMN (MDIPMN); (2) mixed duct IPMN; and (3) branch duct IPMN (BD-IPMN). In a review of a published series of 50 or more cases, the frequency of malignancy was $61.6 \%$ and the frequency of invasive IPMN was $43.1 \%$. Considering these incidences, it was felt that surgical resections were strongly recommended in all fit patients in whom the main duct was dilated greater than $5 \mathrm{~mm}$ in diameter and in those who presented with symptoms of jaundice, pancreatitis, diabetes, and/or steatorrhea [21]. However, much of the controversy surrounds the decision to resect or observe BDIPMN lesions. Tanaka et al. [21] published international consensus guidelines in 2012 for the management of IPMN of the pancreas. The frequency of malignancy in resected
BD-IPMN was 25.5\% (range 6.3-46.5\%); the frequency of invasive cancer was $17.7 \%$ (range $1.4-36.7 \%$ ). This may warrant consideration of resection in many patients; however, BD-IPMN are often discovered incidentally [8] and occur in elderly patients who may have comorbid conditions. Therefore, in these patients, surgery may be indicated when the cyst is greater than $3 \mathrm{~cm}$, imaging follow-up reveals growth, a mural nodule is present, and/or positive cytology is obtained [21]. In the absence of these clinical indications, the consensus group concluded that BD-IPMN lesions less than $3 \mathrm{~cm}$ could be observed, particularly in elderly patients. In comparison, other authors have recommended consideration of enucleation for BD-IPMN [22]. However, the incidence of pancreatic fistula was $43 \%$, and, as such, we have not undertaken enucleation for BD-IPMN. In reviewing our own data (reported previously [23]), preoperative testing did not reliably differentiate main versus branch duct lesions, and thus the decision to observe should be undertaken cautiously. Nevertheless, there does appear to be a role for observation in patients with lesions that are asymptomatic and not showing growth with no mural nodules.

Tanaka et al. also published guidelines for MCN. MCN is defined by the presence of ovarian stroma and has a low prevalence of invasive carcinoma [21]. These tumors often occur in young women, and the risk of progression to invasion, though not accurately known, is possible [24, 25]. Based on this, the consensus in MCN is to resect, which can often be achieved laparoscopically when involving the body of the pancreas [21]. In the frail elderly patient, ablation may be considered [21]. Because of the potential for surgical management, it is important to distinguish mucinous from nonmucinous cystic neoplasms in the preoperative evaluation. The utility of EUS cytology and cyst fluid analysis (CEA and amylase) has previously been assessed and proven to be highly specific for differentiating these tumors, while EUS morphology alone did not distinguish between the two groups [20].

SPN of the pancreas is an entity that occurs mainly in young women in the third decade of life; however, as in our series, others have seen a few cases which occur in men $[26,27]$. These tumors have both solid and cystic elements and can present with solitary lesions in the head, body, and tail of the pancreas, and a certain percentage of patients will present with metastases at the time of diagnoses [26, 27]. Because these lesions occur mainly in young women and have a malignant potential, the general consensus is to resect, depending on the location of the tumor, either by pancreaticoduodenectomy or distal pancreatectomy \pm splenectomy. Cure rates are high, and patients often do well after removal of metastases when they occur in the liver $[26,27]$.

Although our work represents an update on the management of pancreatic cystic neoplasms, it has several limitations. It is a retrospective review of data from one institution and thus has an inevitable selection bias. The ideal study would include data on those patients with pancreatic cystic lesions who do not undergo surgery, which could then serve as a population for comparison. Patients managed nonoperatively, however, are followed up by several types of physicians, often including nonsurgeons, which makes identification of such patients difficult. In addition, some 
are only followed up for a few years and are then released from follow-up. Because of these pitfalls, the overall incidence of pancreatic cystic neoplasms could not be calculated in our study. However, there were approximately 75 patients followed up by surgeons during the second era, based on CT and EUS criteria; in the first era, we operated on all cystic lesions that were proven not to be pseudocysts and were evaluated by a surgeon. The majority of these were serous cystic adenomas, and they are followed up with CT scans every six months for two years and yearly scans for three years. Only one branch duct IPMN became symptomatic during this time period and required surgical intervention.

\section{Conclusions}

In summary, our experience with cystic tumors of the pancreas in the modern era reveals a dramatic change in the indication for surgery, which is mainly related to SCN and IPMN. In the first decade reviewed, SCN was the most commonly resected cystic tumor, and, in the second decade, IPMN was the most commonly resected tumor and SCN was the second least. This is likely related to the advancements in imaging and fluid analysis using EUS. Our present approach to SCN is to surgically resect those that are symptomatic, those that are large, and those that are growing on sequential follow-up imaging. Because of the possibility of a benign MCN undergoing malignant transformation, we recommend resection of these tumors. SPN lesions often occur in young women, can harbor malignancy, and sometimes present with metastases. Therefore, we suggest that resection is indicated in relation to the site of the tumor in the pancreas. These patients may also benefit from metastasectomy. MD-IPMN patients who present with jaundice, pancreatitis, steatorrhea, diabetes, and/or main duct diameter greater than $5 \mathrm{~mm}$ should undergo surgical resection. This may include pancreaticoduodenectomy or a total pancreatectomy. In BD-IPMN, surgery is reserved for those who are symptomatic, those with enlarging lesions on follow-up imaging, those whose cytology reveals atypia, and those with a mural nodule on imaging. For those with mixed type IPMN, surveillance is performed for high risk patients with comorbidities and those who have a borderline dilated duct that is less than $5 \mathrm{~mm}$. However, in those patients who have major pancreatic duct dilatation and have cytology that is atypical, we recommend resection. It is our contention that the proper use of imaging and cyst fluid analysis has led to less surgery in asymptomatic SCN and increased recognition of IPMN where the prevalence of malignancy is a reality.

\section{Conflict of Interests}

The authors declare that there is no conflict of interests regarding the publication of this paper.

\section{Acknowledgment}

The authors would like to acknowledge the funding provided by the Department of Surgery, Loyola University Health System.

\section{References}

[1] T. A. Laffan, K. M. Horton, A. P. Klein et al., "Prevalence of unsuspected pancreatic cysts on MDCT," The American Journal of Roentgenology, vol. 191, no. 3, pp. 802-807, 2008.

[2] K. S. Spinelli, T. E. Fromwiller, R. A. Daniel et al., "Cystic pancreatic neoplasms: observe or operate," Annals of Surgery, vol. 239, no. 5, pp. 651-659, 2004.

[3] C. Fernández-Del Castillo, J. Targarona, S. P. Thayer et al., "Incidental pancreatic cysts: clinicopathologic characteristics and comparison with symptomatic patients," Archives of Surgery, vol. 138, no. 4, pp. 427-434, 2003.

[4] C. M. Pyke, J. A. van Heerden, T. V. Colby, M. G. Sarr, and A. L. Weaver, "The spectrum of serous cystadenoma of the pancreas: clinical, pathologic, and surgical aspects," Annals of Surgery, vol. 215, no. 2, pp. 132-139, 1992.

[5] V. Chaudhary and S. Bano, "Imaging of the pancreas: recent advances," Indian Journal of Endocrinology and Metabolism, vol. 15, no. 5, pp. S25-S32, 2011.

[6] M. K. Sheehan, K. Beck, J. Pickleman et al., "Spectrum of cystic neoplasms of the pancreas and their surgical management," Archives of Surgery, vol. 138, no. 6, pp. 657-662, 2003.

[7] B. G. Turner and W. R. Brugge, "Pancreatic cystic lesions: when to watch, when to operate, and when to ignore," Current Gastroenterology Reports, vol. 12, no. 2, pp. 98-105, 2010.

[8] J. J. Farrell and C. Fernández-Del Castillo, "Pancreatic cystic neoplasms: management and unanswered questions," Gastroenterology, vol. 144, no. 6, pp. 1303-1315, 2013.

[9] N. P. Valsangkar, V. Morales-Oyarvide, S. P. Thayer et al., "851 resected cystic tumors of the pancreas: a 33-year experience at the Massachusetts General Hospital," Surgery, vol. 152, no. 3, pp. S4-S12, 2012.

[10] M. Fukasawa, H. Maguchi, K. Takahashi et al., "Clinical features and natural history of serous cystic neoplasm of the pancreas," Pancreatology, vol. 10, no. 6, pp. 695-701, 2011.

[11] J. C. King, T. T. Ng, S. C. White, G. Cortina, H. A. Reber, and O. J. Hines, "Pancreatic serous cystadenocarcinoma: a case report and review of the literature," Journal of Gastrointestinal Surgery, vol. 13, no. 10, pp. 1864-1868, 2009.

[12] J. F. Tseng, A. L. Warshaw, D. V. Sahani et al., "Serous cystadenoma of the pancreas: tumor growth rates and recommendations for treatment," Annals of Surgery, vol. 242, no. 3, pp. 413421, 2005.

[13] M. T. Yip-Schneider, H. Wu, R. P. Dumas et al., "Vascular endothelial growth factor, a novel and highly accurate pancreatic fluid biomarker for serous pancreatic cysts," Journal of the American College of Surgeons, vol. 218, no. 4, pp. 608-617, 2014.

[14] C. M. Schmidt, "Is surgical intervention for cystic neoplasms of the pancreas being underutilized?" Journal of Gastrointestinal Surgery, vol. 18, no. 1, pp. 184-186, 2014.

[15] H.-J. Lee, M.-J. Kim, J.-Y. Choi, H.-S. Hong, and K. A. Kim, "Relative accuracy of CT and MRI in the differentiation of benign from malignant pancreatic cystic lesions," Clinical Radiology, vol. 66, no. 4, pp. 315-321, 2011.

[16] Y. Nakai, H. Isayama, T. Itoi et al., "Role of endoscopic ultrasonography in pancreatic cystic neoplasms: where do we stand and where will we go?" Digestive Endoscopy, vol. 26, no. 2, pp. 135-143, 2014.

[17] T. Tirkes, K. Sandrasegaran, R. Sanyal et al., "Secretin-enhanced MR cholangiopancreatography: spectrum of findings," Radiographics, vol. 33, no. 7, pp. 1889-1906, 2013. 
[18] M. A. Khashab, K. Kim, A. M. Lennon et al., "Should we do EUS/FNA on patients with pancreatic cysts? the incremental diagnostic yield of EUS over CT/MRI for prediction of cystic neoplasms," Pancreas, vol. 42, no. 4, pp. 717-721, 2013.

[19] K. B. Lewandrowski, J. F. Southern, M. R. Pins, C. C. Compton, and A. L. Warshaw, "Cyst fluid analysis in the differential diagnosis of pancreatic cysts: a comparison of pseudocysts, serous cystadenomas, mucinous cystic neoplasms, and mucinous cystadenocarcinoma," Annals of Surgery, vol. 217, no. 1, pp. 41-47, 1993.

[20] S. Attasaranya, S. Pais, J. LeBlanc, L. McHenry, S. Sherman, and J. M. DeWitt, "Endoscopic ultrasound-guided fine needle aspiration and cyst fluid analysis for pancreatic cysts," Journal of the Pancreas, vol. 8, no. 5, pp. 553-563, 2007.

[21] M. Tanaka, C. Fernández-Del Castillo, V. Adsay et al., "International consensus guidelines 2012 for the management of IPMN and MCN of the pancreas," Pancreatology, vol. 12, no. 3, pp. 183197, 2012.

[22] O. Turrini, C. M. Schmidt, H. A. Pitt et al., "Side-branch intraductal papillary mucinous neoplasms of the pancreatic head/uncinate: resection or enucleation?" $H P B$, vol. 13 , no. 2 , pp. 126-131, 2011.

[23] J. K. Plichta, K. Ban, Z. Fridirici et al., "Should all branch-duct intraductal papillary mucinous neoplasms be resected?" The American Journal of Surgery, vol. 209, no. 3, pp. 478-482, 2015.

[24] M. G. Sarr, H. A. Carpenter, L. P. Prabhakar et al., "Clinical and pathologic correlation of 84 mucinous cystic neoplasms of the pancreas. Can one reliably differentiate benign from malignant (or premalignant) neoplasms?” Annals of Surgery, vol. 231, no. 2, pp. 205-212, 2000.

[25] R. E. Jimenez, A. L. Warshaw, K. Z'graggen et al., "Sequential accumulation of K-ras mutations and p53 overexpression in the progression of pancreatic mucinous cystic neoplasms to malignancy," Annals of Surgery, vol. 230, no. 4, pp. 501-511, 1999.

[26] S. Reddy, J. L. Cameron, J. Scudiere et al., "Surgical management of solid-pseudopapillary neoplasms of the pancreas (Franz or Hamoudi tumors): a large single-institutional series," Journal of the American College of Surgeons, vol. 208, no. 5, pp. 950-957, 2009.

[27] J. M. Butte, M. F. Brennan, M. Gönen et al., "Solid pseudopapillary tumors of the pancreas. Clinical features, surgical outcomes, and long-term survival in 45 consecutive patients from a single center," Journal of Gastrointestinal Surgery, vol. 15, no. 2, pp. 350-357, 2011. 


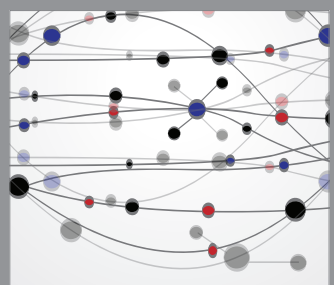

The Scientific World Journal
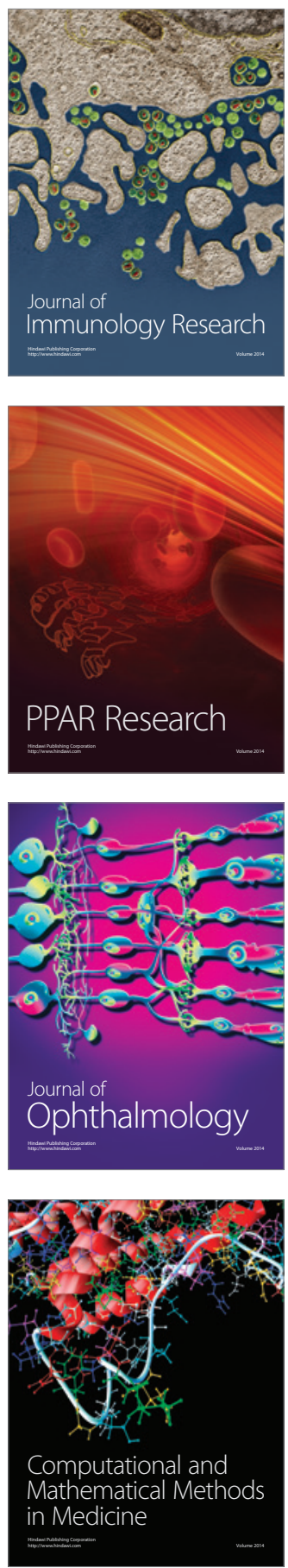

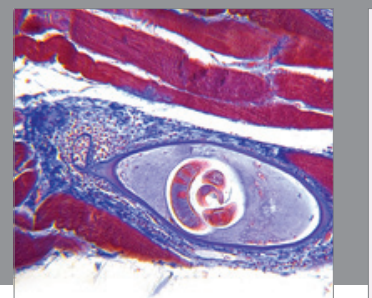

Gastroenterology

Research and Practice
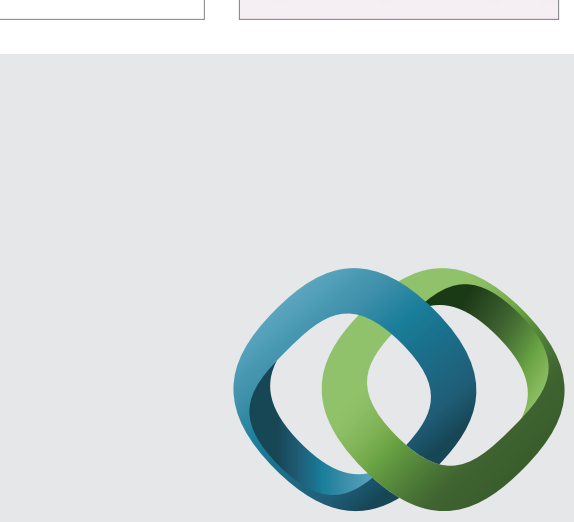

\section{Hindawi}

Submit your manuscripts at

http://www.hindawi.com
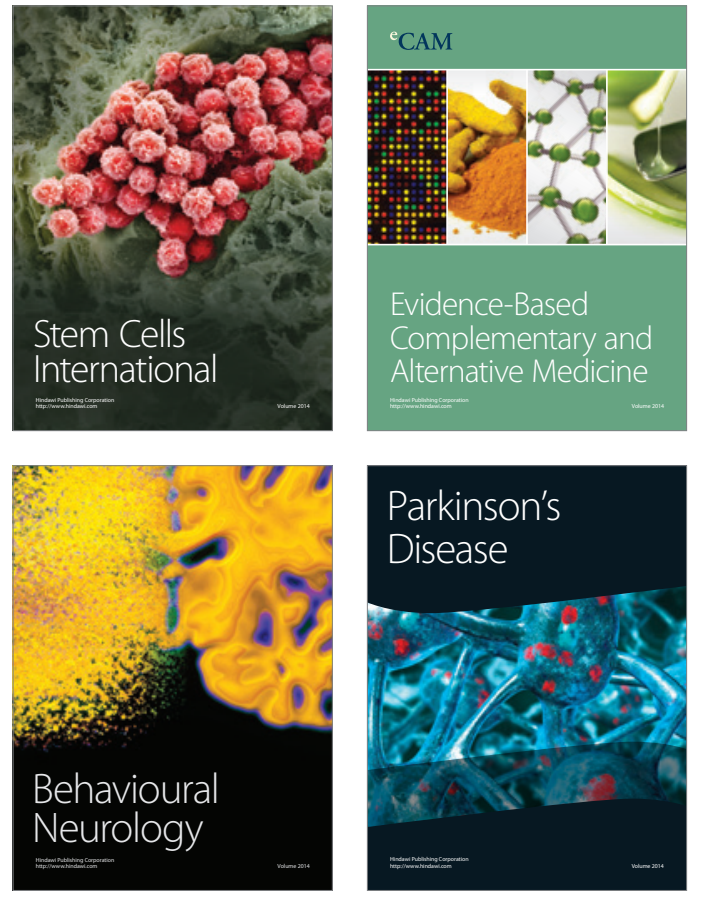
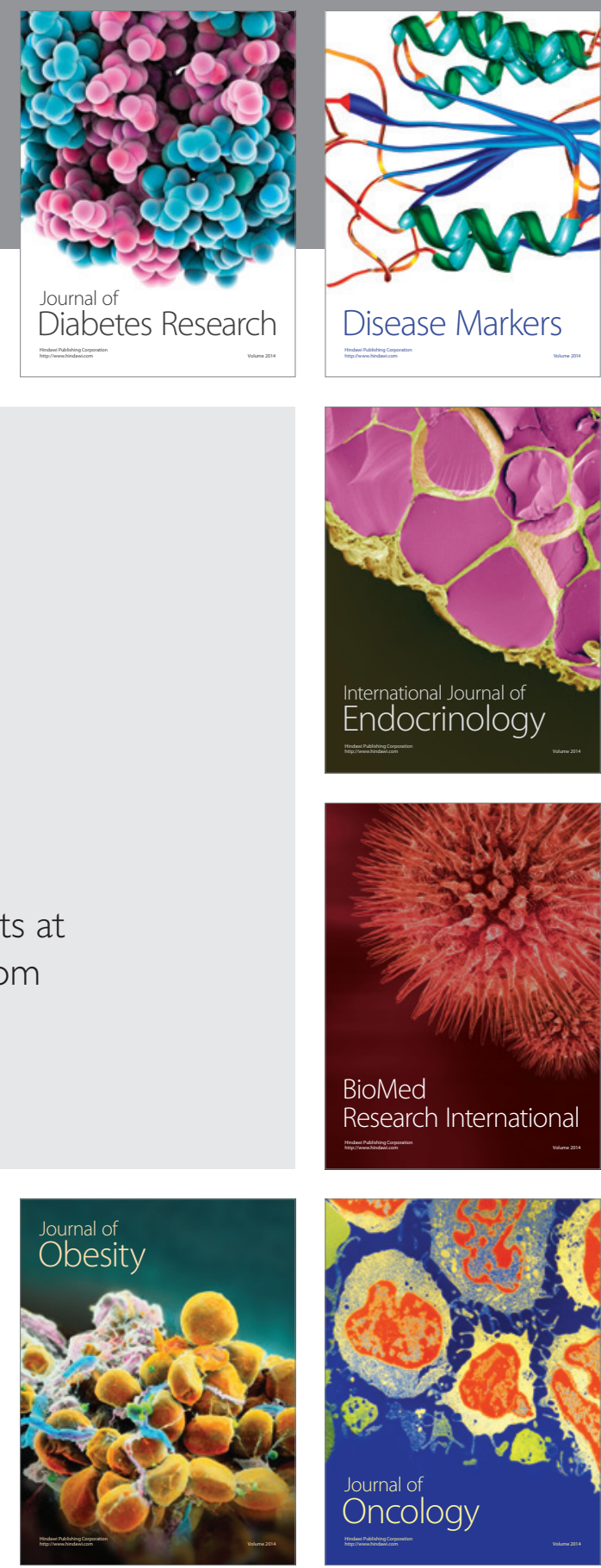

Disease Markers
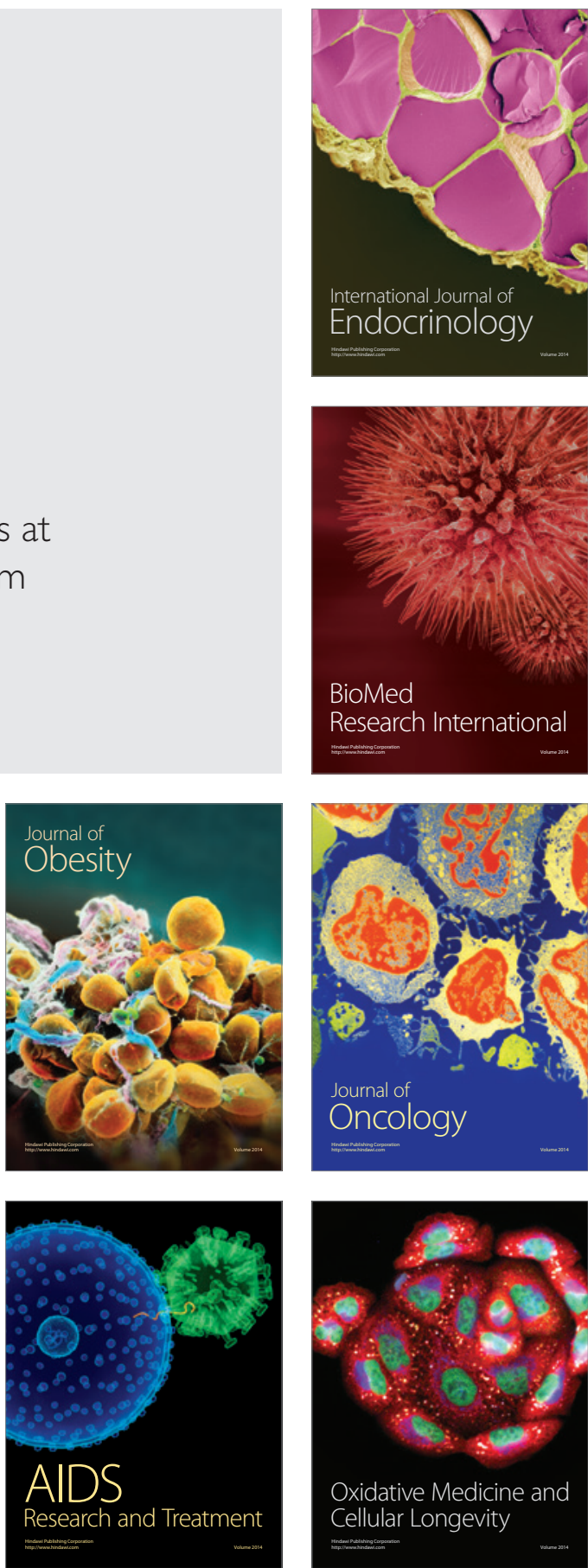